\title{
Building Mathematical Fluency for Students with Disabilities or Students At-Risk for Mathematics Failure
}

\author{
Vanessa Hinton $^{1 *}$, Shaunita D. Strozier ${ }^{2}$, Margaret M. Flores ${ }^{3}$ \\ ${ }^{1}$ Auburn University \\ ${ }^{2}$ Valdosta State University \\ ${ }^{3}$ Auburn University
}

\begin{abstract}
It is incredibly important for students who are at-risk for mathematics failure or who have a disability which hinders mathematical performance to improve in their mathematical achievement. One way to improve mathematical achievement is through building fluency in mathematics. Fluency in mathematics is the ability to solve problems automatically and with accuracy. One method of building fluency for students who are at-risk or who have a disability includes the concrete-representational-abstract sequence of instruction that is paired with strategic instruction (CRA-SI). A brief overview is provided on CRA-SI instruction along with a review of the literature that shows CRA-SI instruction as effective instruction for students who are at-risk or who have a disability.
\end{abstract}

Key words: Mathematical Fluency, Disabilities, Students At-Risk.

\section{Introduction}

Mathematical achievement of individuals strongly predicts their success later in life (Rivera-Batiz, 1992). To create a solid foundation of mathematical achievement, students need to build fluency in solving mathematical problems (National Mathematical Advisory Panel, 2008). Mathematics fluency is the ability to solve problems accurately and automatically (National Mathematical Advisory Panel, 2008). Fluency in problem solving, whole numbers and fractions have to be achieved for students to solve problems that require several steps and move to higher order thinking in mathematics (National Mathematical Advisory Panel, 2008). Fluency solving whole numbers and fractions is a critical skill, and it can be very difficult for some students to obtain, especially students who are considered at-risk for mathematical difficulties or who have a disability. One way of providing effective instruction that builds mathematical fluency in whole numbers and fractions (e.g., solving problems with whole numbers and fractions accurately and automatically) for diverse groups of students is through a mathematic sequence of instruction referred to as concrete- representational- abstract (CRA). CRA is often coupled with strategic instruction (CRA-SI; Miller, 2009). The purpose of this article is to provide an overview of CRA-SI instruction that is used to build mathematical fluency in solving computational problems using whole numbers and fractions.

Miller (2009) explains CRA-SI builds conceptual, procedural, and declarative mathematical knowledge. Conceptual knowledge is an understanding of the mathematical concept itself (Miller, 2009). Procedural knowledge is an understanding of the steps required to solve the problem (Miller, 2009). Declarative knowledge is the ability to solve problems accurately and automatically which is also referred to as fluency in mathematics (Miller, 2009).

CRA-SI instruction involves three phases and is effective in teaching computation of whole numbers and fractions because it involves visual, auditory and kinesthetic learning which has been shown to reach a variety of students who learn using different modalities (Witzel, 2005). The first phase of the CRA-SI is the concrete phase. The concrete phase of the instructional sequence requires the teacher and students to solve problems using objects. During the concrete phase; students see, hear, and move objects to demonstrate what is happening with the numbers as well as the procedures to solve the problems. When implementing instruction in

* Corresponding Author: Vanessa Hinton,vmh0002@auburn.edu 
the concrete phase, the teacher begins the lesson with an advanced organizer in which he or she tells students what they will learn and makes the lesson meaningful to the students (Flores, 2009; Kaffer \& Miller, 2011; Miller, 2009). Next, the teacher demonstrates solving mathematics problems (Flores, 2009; Kaffer \& Miller, 2011; Miller, 2009). This is also called modeling. When modeling, the instructor shows students what is happening with the numbers as well as mathematical procedures or steps to solve the problems. After modeling, guided practice is implemented (Flores, 2009; Kaffer \& Miller, 2011; Miller, 2009). Guided practice is when the teacher and students demonstrate what is happening with the numbers and solve the mathematical problems together. During guided practice, the teacher gives students feedback and helps students solve problems as they develop an understanding of the mathematical concept. After guided practice, students solve problems independently which is called independent practice (Flores, 2009; Kaffer \& Miller, 2011; Miller, 2009). Independent practice requires students to solve problems on their own without teacher assistance. During independent practice, the teacher monitors students' understanding and provides productive feedback regarding students' responses. The last step is the post organizer (Flores, 2009; Kaffer \& Miller, 2011; Miller, 2009). The post organizer involves the teacher prompting students to reflect and demonstrate what they have learned.

The second phase of CRA-SI is the representative phase. The instructor teaches the same concept that was illustrated in the concrete phase and the same type of mathematical problems; except instruction occurs through the use of drawings instead of the manipulation of objects. The representative phase acts as a bridge between solving problems using objects and solving problems using numbers only (Flores, 2009). The representative phase of instruction involves an advance organizer, modeling, guided practice, independent practice, and a post organizer as well (Flores, 2009; Kaffer \& Miller, 2011; Miller, 2009). In the advanced organizer, the teacher tells students what they will learn and makes the lesson meaningful to the students. Next is the demonstration or modeling step, and in this step the teacher models solving the mathematics problem (Flores, 2009; Kaffer \& Miller, 2011; Miller, 2009). When modeling, the instructor shows students what is happening with the numbers as well as the mathematical procedures. After the demonstration step, guided practice is implemented. Guided practice is when the teacher and students demonstrate what is happening with the numbers and solve the mathematical problems together (Flores, 2009; Kaffer \& Miller, 2011; Miller, 2009). During guided practice, the teacher gives students feedback and helps students develop an understanding of the mathematical concept. The fourth step is independent practice. Independent practice requires students to solve problems on their own without teacher assistance (Flores, 2009; Kaffer \& Miller, 2011; Miller, 2009). During independent practice, the teacher monitors students' understanding and gives students feedback based on their responses. The last step is the post organizer. The post organizer involves the teacher asking students to reflect and demonstrate what they have learned (Flores, 2009; Kaffer \& Miller, 2011; Miller, 2009).

The last phase of CRA-SI is the abstract phase. In the abstract phase teachers and students solve mathematical problems using numbers only; therefore they do not use objects or pictures (Flores, 2009; Kaffer \& Miller, 2011; Miller, 2009). Before instruction starts in the abstract phase, strategy instruction is implemented, and students are taught a strategy that guides the student in remembering the procedures to solve the mathematical problem. Teaching students a strategy that helps them remember information or helps students apply information is called strategy instruction (Horowitz, N.d). Horowitz (N.d) explains that strategies are useful for students when they learn new skills and when they have to apply the skills. In CRA-SI, the strategy usually involves a mnemonic that helps students remember the steps or procedures in solving the mathematical problems (Flores, 2009; Kaffer \& Miller, 2011; Miller, 2009). Mnemonics used as a strategy in CRA-SI are DRAW, RENAME, FASTDRAW, STAR, and SUMLOW (Flores, 2010; Harris, Miller, \& Mercer, 1995; Kaffar \& Miller, 2011; Maccini \& Hughes, 2000; Mercer \& Miller, 1992; Strozier, Flores, Shippen, \& Hinton, 2012; Witzel, Mercer, \& Miller, 2003). These strategies will be discussed in more depth later in the manuscript.

When implementing instruction during the abstract phase of CRA-SI, the teacher will provide an advanced organizer, demonstration or modeling session, guided practice, independent practice, and post organizer (Miller, 2009). The advanced organizer is when the teacher tells students what they will learn and makes the lesson relevant to the students (Flores, 2009; Kaffer \& Miller, 2011; Miller, 2009). The next step is called the demonstration or modeling session, and the instructor shows students what is happening with the numbers as well as mathematical procedures. After the demonstration or modeling session, the teacher implements guided practice (Flores, 2009; Kaffer \& Miller, 2011; Miller, 2009). Guided practice is when the teacher and students solve the mathematical problems together (Flores, 2009; Kaffer \& Miller, 2011; Miller, 2009). During guided practice, the teacher gives helps students build an understanding of the mathematical concept. The fourth step is independent practice. During independent practice students solve problems without teacher assistance; however, the teacher monitors students' understanding and provides feedback. Lastly, the teacher implements the post organizer. The post organizer involves the teacher directing students to reflect and show what they 
have learned. This article explores the research on CRA-SI instruction which is used to build mathematical fluency in solving computational problems using whole numbers and fractions.

\section{Method}

This article reviews the literature for CRA-SI supplemental instruction that has been shown effective in building fluency in mathematics (e.g., the ability to solve computational problems using whole numbers and fractions accurately and automatically; National Mathematical Advisory Panel, 2008). A computer search of ERIC and PsycINFO was used to locate studies published between 1988 to 2013. The timeframe was selected because the initial research on the CRA-SI was conducted in 1988 by Peterson, Mercer, and O'Shea. Key descriptors or root word descriptors (concrete, semi-concrete, representational, abstract, at-risk, mathematical difficulties, special education) were used in combination with key mathematic descriptors or root forms of descriptors (mathematic interventions, mathematic instruction, supplemental instruction, explicit instruction, mathematics) to identify possible articles. The criteria for selecting studies were as follows: (a) the research is applicable to fluency in mathematics, (b) the research had to examine the CRA-SI instruction, (c) the research had to focus on student performance outcomes as a dependent variable, and (d) the research had to be published in a peer reviewed journal and written in English. All four criteria had to be fulfilled to meet the scope of the literature review.

Researchers show CRA-SI as a beneficial instructional method for teaching students with diverse backgrounds, students with disabilities such as specific learning disabilities, intellectual disabilities, and emotional disabilities as well as students who are at-risk for mathematics failure (Butler, Miller, Crehan, Babbitt \& Pierce, 2003; Flores, 2009; Peterson, Mercer, O’Shea, 1988; Maccini \& Hughes, 2000; Mercer \& Miller, 1992; Morin \& Miller, 1998; Strozier, Flores, Shippen, \& Hinton, 2012; Witzel, Mercer, \& Miller, 2003). Research has shown that CRA-SI is effective in building mathematical fluency with place value, addition and subtraction of numbers, multiplication, regrouping, fractions, integers and algebra (Bulter et al., 2003, Flores, 2010; Peterson et al., 1988; Maccini \& Hughes, 2000; Mercer \& Miller, 1992; Morin \& Miller, 1998; Strozier et al., 2012; Witzel et al., 2003). A summary of the research studies chronicled by date is provided in Table 1 . The studies are described in the manuscript by skill area and are as follows (a) mathematics fluency in place value, addition, and subtraction; (b) mathematics fluency in multiplication; (c) mathematics fluency in regrouping in addition, subtraction, and multiplication; and (d) mathematics fluency in fractions, integers, and algebra.

Table 1.CRA-SI studies for mathematics fluency by date

\begin{tabular}{llll}
\hline \multicolumn{1}{c}{ Authors and Date } & \multicolumn{1}{c}{ Intervention } & \multicolumn{1}{c}{ Participants } & \multicolumn{1}{c}{ Design } \\
\hline Peterson, S. K., & CRA sequence & 24 (20 males \& 4 & Group comparison of Place value \\
Mercer, C. D., \& & & females) ranging in & CRA sequence to \\
O'Shea, L (1988) & & age from 8 to 13 & traditional \\
& & with specific & instruction using \\
& & learning disabilities & only numbers
\end{tabular}

Mercer, C. D. \& Miller, S. P (1992)
CRA sequence with DRAW
109 elementary school students (102 with specific learning disabilities, 5 with emotional disorder, and 2 - at risk)

Harris, C. A., Miller, CRA sequence S. P., \& Mercer, C. with DRAW and D. (1995) FAST DRAW

Morin, V. A., \& Miller, S. P (1998)
CRA sequence with DRAW and FAST DRAW
99 second graders and 13 second graders with disabilities Three seventh grade students with intellectual disabilities
Place value and basic facts
Multiple baseline Multiplication across settings

Multiple baseline across subjects
Multiplication and related word problems 
Table 1 (continued) CRA-SI studies for mathematics fluency by date

\begin{tabular}{|c|c|c|c|c|}
\hline Authors and Date & Intervention & Participants & Design & Skill Area \\
\hline $\begin{array}{l}\text { Maccini, P. \& } \\
\text { Hughes, C. (2000) }\end{array}$ & $\begin{array}{l}\text { CRA sequence with } \\
\text { STAR }\end{array}$ & $\begin{array}{l}\text { Six students with } \\
\text { specific learning } \\
\text { disability }\end{array}$ & $\begin{array}{l}\text { Multiple baseline } \\
\text { across subjects }\end{array}$ & $\begin{array}{l}\text { Word problems } \\
\text { involving integers }\end{array}$ \\
\hline
\end{tabular}

Witzel, B. S., Mercer, CRA Sequence

C. D., \& Miller, M. D with STAR (2003)

Flores, Margaret (2009)

Flores, Margaret (2010)

Kaffar, B. J. \& Miller, S. P. (2011) DRAW DRAW RENAME
CRA sequence with

CRA sequence with Six third graders at

CRA Sequence and

Strozier, S. D. Flores, M., Shippen M. \& Hinton, V. (2012)

Flores, M. M.,

Hinton, V., Strozier, S. D., \& Terry, L. (in press)

Flores, M. M., CRA Sequence and S. D., (in press)
Three students at risk for mathematics failure
358 sixth and seventh graders (34 students with disabilities or at risk) risk for mathematics failure

24 students at-risk for mathematics failure
Group comparison of Algebra CRA-SI to abstract only

Multiple probe across Subtractions with subjects

Multiple probe across Subtractions with subjects regrouping to the tens and hundreds place

Subtraction with regrouping abstract instruction

CRA sequence and Two $8^{\text {th }}$ grade SUMLOWS CRA Sequence and DRAW Twe (one with specific learning disability and one at risk)

11 students with autism spectrum disorder, intellectual disability, or developmental delay

RENAME
Multiple probe across behaviors

One-step, two-step, and multiple step algebraic equations and a specific mnemonic for solving equation

Pre and posttest measures and comparison to established growth rates

Multiple probe across Subtraction with behaviors regrouping and multiplication with regrouping

\section{CRA-SI for Place Value, Addition and Subtraction Fluency}

Peterson, Mercer, and O'Shea (1988) conducted the initial research regarding CRA. Students who received instruction were eligible for special education services under the category of specific learning disability (SLD). Peterson et al. compared the effects of the CRA instructional sequence to instruction that involve numbers only regarding students' acquisition, maintenance, retention, and generalization of place value skills. There were 24 participants total who were in elementary or middle school ranging in age from 8 to 13 and they received mathematics instruction in special education classrooms. Results show that the CRA teaching sequence was more effective than instruction with numbers only for teaching place value skills to students with SLD. Specifically students who learned place value with the use of manipulatives and pictorial representations before using numbers only had higher rates of skill acquisition, maintenance, and retention.

Mercer and Miller (1992) extended the research conducted by Peterson et al, and created the Strategic Math Series curriculum to teach place value and addition and subtraction fact fluency to students who either received special education services or who were at-risk for mathematics failure. Strategic instruction included the DRAW strategy to help students remember procedures in solving the problems. The DRAW strategy consisted 
of four steps and the mnemonic DRAW to help students remember each step. When using DRAW students were to a) Discover the sign, b) Read the problem, c) Answer or DRAW a conceptual representation of the problem using lines and tallies, and d) Write the answer and check.

There were 109 participants in the study and the research design included a pre and posttest. Students were given tests on place value, addition facts, and subtraction facts before CRA-SI instruction started and then tested after students finished the CRA-SI curriculum for each skill. Overall, Miller and Mercer concluded that the field test data indicated that students with learning difficulty were able to (a) acquire computational skills across facts, (b) solve word problems with and without extraneous information, (c) create word problems involving facts, (d) apply a mnemonic strategy to difficult problems, (e) increase rate of computation, and (f) generalize math skills across examiners, settings, and tasks. CRA continues to hold promise for teaching place value and basic facts in addition and subtraction.

\section{CRA-SI for Multiplication Fluency}

Harris, Miller, and Mercer (1995) evaluated the effectiveness of teaching multiplication skills to elementary students with disabilities in the general education classrooms. Students learned the DRAW and FAST DRAW strategy to help them remember mathematical procedures. DRAW was used to help students remember steps to solve multiplication facts. FAST DRAW was used to help students remember the steps in solving word problems with multiplication. FAST stands for Find what you're looking for, Ask yourself, "What are the parts of the problem?" Set up the numbers, and Tie down the sign. DRAW in the mnemonic strategy stands for Discover the sign, Read the problem, Answer or DRAW a conceptual representation of the problem using lines and tallies, and check, and Write the answer and check.

To examine the effectiveness of CRA and the strategy of FAST DRAW, Harris et al used a single-subject multiple baseline design across three functionally independent, yet similar, classrooms. Participants of this study included 13 second graders with disabilities and 99 second graders without disabilities. Teachers involved in this study attended a training session to familiarize themselves with the teaching procedures of CRA-SI instruction. Comparison of median rate scores during baseline and the final phase of instruction show that the average amount of change was an increase of 8.4 correct digits and a decrease of 21.3 incorrect digits per minute. This study showed when given a strategy for counting objects within groups, students with disabilities were as successful as their peers without disabilities at reaching correct answers on the independent practice sections of the daily worksheets.

Morin and Miller (1998) evaluated the effectiveness of teaching multiplication facts and related word problems using CRA-SI for three middle school students with intellectual disabilities. The participants were middle school students with severe intellectual disabilities. The participants' ages ranged from 15 to 16 years old. Similar to Harris et al DRAW and FAST DRAW were the strategies taught to help students remember steps to solve multiplication facts and the steps to solve word problems. Participants were referred to as Student One, Student Two, and Student Three.

A single-subject multiple baseline design across participants was used to demonstrate experimental control and document the effects of the intervention. Baseline and intervention data along with pre-and posttest scores reflected significant progress for all three subjects. Student One earned 50 percent on her pretest and 90 percent on her posttest. Student Two earned 70 percent on the pretest and 90 percent on the posttest. Student Three earn 20 percent on the pretest and 90 percent on the posttest. The percent gain scores for Students One, Two, and Three were 40, 20, and 70 points respectively. The findings of this investigation suggest that students with severe intellectual disabilities can learn to solve multiplication facts and related word problems using CRA-SI instructional procedures.

Flores, Hinton, Strozier, and Terry (in press) examined CRA-SI in building fluency in addition and subtraction facts for students who have autism spectrum disorders, intellectual disability, and developmental delay. The DRAW strategy was implemented to help students remember steps in solving the problems. A pretest and posttest design was used to evaluate the effects of CRA-SI. Eleven students participated in the study. The students ranged in age from five to twelve years of age. Nine students received instruction in addition facts zero through nine and two students received instruction in subtraction facts zero through nine. Results indicated significant improvement in students' computation of addition or subtraction facts and in students' growth rates which were compared to growth rates established by Fuchs and Fuchs (1993). 


\section{CRA-SI for Regrouping Fluency in Addition, Subtraction and Multiplication}

Flores $(2009,2010)$ extended the CRA-SI line of research to include subtraction with regrouping. This study investigated the effectiveness of the CRA sequence on the computation performance of students with specific learning disabilities and students identified as at risk for failure in mathematics. The DRAW strategy was used to help students remember steps in solving problems. The participants in the 2009 study were six third grade students who were failing mathematics in terms of grades and performance on benchmark assessments. Students were given pseudo names of Art, Beto, Ed, Mari, Juan, and May.

A multiple-probe across participants design was used to evaluate the effectiveness of CRA-SI for teaching subtraction with regrouping. All participants showed steady improvement for subtraction with regrouping except for May. May demonstrated some variability in the first four data points after the CRA-SI instructional was implemented, but then she demonstrated a steady upward path indicating improvement. Data were collected four weeks after instruction to see if participants maintained their skill. Five of the six participants maintained performance at or above the criterion level. The results of this study showed a functional relation between CRA-SI instruction and the students' performance on subtraction with regrouping skills.

The 2010 study was a little different because it included subtraction problems with larger numbers (i.e., 0-999) and required grouping in the tens and ones place. Subtraction with regrouping was taught to six students in the third grade who were identified as at-risk for mathematics failure. Similar to the 2009 study, the strategy DRAW was used to help students remember steps to solve the problems. Students in this study were given pseudo names of Ray, Al, Walt, Ron, Joe, and Ann. A multiple-probe across participants design was used to evaluate the efficacy of the CRA-SI for teaching subtraction with regrouping to the tens and hundreds place. All participants showed steady improvement for subtraction with regrouping in the tens and ones place.

Kaffar and Miller (2011) investigated the effects of CRA-SI instruction but included the strategy RENAME as the mnemonic instead of DRAW for subtraction with regrouping. The RENAME mnemonic was made up of six steps. They were (a) Read the problem, (b) Examine the ones, (c) Note the ones, (d) Address the tens column, (e) Mark the tens column, and (f) Examine and not the hundreds and exit with a quick check. The research design was a comparison of instructional methods. Twelve participants received CRA-SI instruction and twelve participants received instruction with numbers only. All participants in both groups were identified as at-risk for mathematics failure. Both groups made gains in subtraction with regrouping, however, the gains made by the students who received instruction with the CRA-SI was greater. Students' mean percentage computation scores increased from $49 \%$ to $90 \%$ for the CRA-SI group while the comparison group's scores increased from $66 \%$ to $72 \%$.

Flores, Hinton, and Strozier (in press) used CRA-SI to teach students who were at-risk for mathematics difficulty how to regroup when subtraction, and multiplication problems. RENAME was the strategy used to help student remember the steps in solving problems. There were three participants who received instruction for subtraction with regrouping and multiplication with regrouping. The design was a multiple probe across behaviors. The behaviors were subtraction with regrouping for the ones place, subtraction with regrouping for the tens and ones place, multiplication with regrouping with a one-digit multiplier, and multiplication with regrouping for a two-digit multiplier.

Al, Lena, and May were pseudo names used for the participants. For the behavior of subtraction with regrouping for the ones place, Al showed improvement from an average of 11 correct digits to 26, Lena showed improvement from an average of 0 correct digits to 21, and May showed improvement from an average of 2 correct digits to 24 . For the behavior of subtraction with regrouping for the tens and ones place, Al showed improvement from an average of 4 correct digits to 21 , Lena showed improvement from an average of 10 correct digits to 36, and May showed improvement from an average of 11 correct digits to 21. For the behavior of multiplication with regrouping with a one-digit multiplier, Al showed improvement from an average of 0 correct digits to 42, Lena showed improvement from an average of 0 correct digits to 25, and May showed improvement from an average of 5 correct digits to 24. For the behavior of multiplication with regrouping with a two-digit multiplier, Al showed improvement from an average of 0 correct digits to 24. Lena and May did not receive instruction for multiplication with a two-digit multiplier because the school year ended before instruction could begin. 


\section{CRA-SI for Fluency in Fractions, Integers and Algebra}

CRA-SI instruction was demonstrated to be successful in teaching skills such as solving problems with fractions, integers, and algebra concepts at the secondary level for students with disabilities or who are at-risk for mathematics failure. The study conducted by Butler, Miller, Crehan, Babbitt, and Pierce (2003) taught middle school students with mild or moderate disabilities equivalent fraction concepts. The design of the study included a comparison of instructional methods. The researchers compared the effects of CRA-SI to instructional that only involved representational and abstract instruction (RA). Instead of learning a strategy with a specific mnemonic such as DRAW or RENAME, students were provided cue cards to use as their strategy to remember the steps in solving problems. This extension of the literature was significant because the researchers investigated the need of students with mild and moderate disabilities to receive concrete level instruction.

Participants in this study were 50 students with mild and moderate disabilities. These participants ranged in age from eleven to fifteen and were enrolled in grades six, seven and eight. Participants in this study were split into two treatment groups. Twenty-six students formed the CRA-SI group, and 24 students formed the RA group. The majority of these students (i.e., 42) were identified with a specific learning disability in mathematics. All students received mathematics instruction in a resource room setting. In this study, an additional 65 students enrolled in the general education class took the post assessment only. The information obtained from this comparison group was used was used to give an estimate of what a student without a disability understands about fractions by the end of the eighth grade year.

Pretests and posttests were used as instruments in this study, consisting of five subtests from the Brigance Comprehensive Inventory of Basic Skills-Revised (CIBS-R) which included concepts such as quantity fractions, area fractions, and abstract fractions. Two additional subtests were designed by the investigator in order to measure skills not assessed in the CIBS-R, such as fraction word problems and improper fractions which had significant correlations with the subtests taken from the CIBS-R. Also, students' attitude toward instruction was measured by an investigator-constructed questionnaire using a three-point Likert scale.

Paired sample t-tests were used to show differences between the pre-assessment and post-assessment scores for both groups. Results showed significant improvements on all measures except for the Area Fractions subtest. A statistically significant difference for the quantity fractions subtest was found which showed students who received concrete level instruction had higher scores on average. Results also showed significant differences between the groups favoring those who received concrete instruction regarding improper fractions and word problems. No differences in attitudes toward instruction were found.

Maccini and Ruhl (2000) investigated CRA-SI using the strategy STAR in solving problems that involve subtraction of integers. The mnemonic device "STAR" prompted students to perform steps to solve subtraction of integers. The steps of "STAR" strategy were (a) Search the word problem, (b) Translate words into a mathematical equation, (c) Answer the problem, and (d) Review the solution. Maccini and Ruhl used a multiple-probe across subjects research design. There were three participants in middle school and diagnosed with a specific learning disability. Maccini and Ruhl examined students' percent of strategy use, percent correct on problem representation, percent correct on problem solution and answer, and generalization. All participants increased their percent of strategy use from baseline to instructional phases in subtraction of integers.

Maccini and Hughes (2000) used CRA-SI instruction to teach subtraction, multiplication, and division of word problems involving integer numbers. Similar to the study of Maccini and Ruhl, the STAR strategy was used to help students remember the steps in solving the problems. The participants were six students with specific learning disability. The researchers examined improvements in (a) searching the word problem, (b) translating the word problem into an equation, (c) identifying the correct operation or operations, (d) drawing a picture of the problem, (e) writing a correct equation, and (f) answering the problems. Results of this study provided initial evidence that students with learning disabilities can be taught to represent word problems involving integer numbers through manipulatives and pictorial representations.

Researchers expanded the use of CRA-SI instruction developed by Maccini and Hughes to teach algebra. Specifically, Witzel, Mercer, and Miller (2003) investigated the effectiveness of the CRA-SI for solving singlevariable equations and solving for a single variable in multiple-variable equations. There were 358 sixth and seventh grade students who participated in the study. Of the 358 participants, 34 students had a disability or were at risk for algebra difficulty. The 34 students who had documented disabilities were in the treatment group. 
The research design was a comparison of instructional methods. A comparison of groups with random assignment of clusters was used in this study. Pretest, posttest, and follow-up measures were collected to measure student performance. Participants in the comparison group received intervention that included explicit instruction techniques and the treatment group received CRA-SI instruction using STAR. Both groups had significant improvements in answering single-variable algebraic equations from the pretest to the posttest and from the pretest to the follow-up. However, students who participated in CRA-SI instruction outperformed students who received only explicit instruction.

Strozier, Flores, Shippen and Hinton (2012) conducted a study examining CRA-SI instruction and a strategy that used the mnemonic SUMLOWS to solve algebra equations. The SUMLOWS mnemonic was made up of seven steps. They were (a) Separate the sides, (b) Unite the like terms, (c) Modify (rewrite) the new equation, (d) Loop (circle) around the coefficient (e) Opposite sign (inverse operation), (f) "What you do on one side, you must do to the other", and (g) Substitute the solution for the variable check.

The research design was a multiple probe across three behaviors. The behaviors were solving one-step equations, solving two-step equations and solving multi-step equations. There were two participants who participated in the study. Pseudo names of Sharon and Clint were given to the participants. Sharon was in the eighth grade, thirteen years old and was identified as having a specific learning disability. Clint was in the eighth grade, thirteen years old and was identified as being at-risk for mathematics failure in algebra. For the behavior of solving one-step algebra equations, Sharon showed improvement from an average of 0 problems correct to 7.5 correct, and Clint showed improvement from an average of 0 problems correct to 7.7 correct. For the behavior of solving two-step algebra equations, Sharon showed improvement from an average of 0 problems correct to 8.4 correct, and Clint improvement from an average of 0 problems correct to 8.3 correct. For the behavior of solving multiple-step equations, Sharon showed improvement from an average of 0 problems correct to 7.2 correct and Clint improvement from an average of 0 problems correct to 7.4 correct.

\section{Conclusion}

CRA-SI includes three phases with each phase using explicit instruction. The three phases are the concrete level which involves objects, representational level which involves drawings, and abstract level which involves numbers only. The three phases of instruction build conceptual knowledge which is an understanding of what is happening with the numbers, procedural knowledge which is an understanding of how to solve the problems, and declarative knowledge which is the ability to solve the problems accurately and automatically. Before students move to the abstract level of instruction, they learn a strategy that helps them remember each step required to solve the problems. Usually the strategy involves a mnemonic. Mnemonics used to prompt students in remembering each step include DRAW, FAST DRAW, RENAME, STAR, and SUMLOWS. DRAW and FAST DRAW have been used to help students remember steps to solve basic addition, subtraction, and multiplication problems. DRAW and RENAME have been used to help students remember steps to solve addition, subtraction, and multiplication problems that involve regrouping. STAR and SUMLOWS have been used to help students remember steps to solve problems that involve integers and algebra. Even though a mnemonic is typically used as a strategy to help students remember steps, cue cards have been used as a strategy students implemented to solve fractions.

CRA-SI instruction has been shown to improve fluency in mathematics. CRA-SI instruction has been used to teach fluency for problems that involve place value, addition and subtraction of numbers, multiplication, regrouping, fractions, integers and algebra. Research has also demonstrated CRA-SI as a beneficial instructional method for teaching students who are at-risk for mathematics failure. This includes instruction that has been provided for students with disabilities such as specific learning disabilities, intellectual disabilities, autism spectrum disorder, developmentally delayed, and emotional disabilities.

\section{References}

Butler, F. C., Miller, S. P.,Crehan,K., Babbitt, B., \& Pierce, T. (2003). Fraction instruction for students with mathematics disabilities: Comparing two teaching sequences. Learning Disabilities Research and Practice, 18, 99-111.

Flores, M. M. (2009). Teaching subtraction with regrouping to students experiencing difficulty in mathematics. Preventing School Failure, 53(3), 145-152. 
Flores, M. M. (2010). Using the concrete-representational-abstract sequence to teacher subtraction with regrouping to students at risk for failure. Remedial and Special Education, 31(3), 195-207.

Flores, M. M., Hinton, V. \& Stroizer, S. (in press) Teaching subtraction and multiplication with regrouping using the concrete-representational-abstract sequence and strategy instruction Learning Disability Research and Practice.

Flores, M. M, Hinton, V. Stroizer, S., \& Terry, L. (in press) Using the concrete-representational- abstract sequence and the strategic instruction model to teach computation to students with autism spectrum disorders and developmental disabilities. Education and Training in Developmental Disabilities.

Harris, C. A., Miller, S. P., \& Mercer, C. D. (1995). The initial multiplication skills to students with disabilities in general education classrooms. Learning Disabilities Research \& Practice, 10(3), 180-195.

Horowitz, S. H. (N.d). Tips to become a strategic learner. Retrieved February 2013 from: The National Center for Learning Disabilities: http://www.ncld.org/students-disabilities/homework-study-skills/becomingstrategic-learner

Kaffer, B. J., \& Miller, S. P. (2011). Investigating the effects of the RENAME Strategy for developing subtraction with regrouping competence among third-grade students with mathematics difficulties. Investigations in Math Learning.

Maccini, P., \& Hughes, C. A. (2000). Effects of a problem-solving strategy on the introductory algebra performance of secondary students with learning disabilities. Learning Disabilities Research \& Practice, 15, 10-21.

Maccini, P., \& Ruhl, K. L. (2000). Effects of graduated instructional sequence on the algebraic subtraction of integers by secondary students with learning disabilities. Education and Treatment of Children, 23, 465-489.

Mercer, C. D., \& Miller, S. P. (1992). Teaching students with learning problems in math to acquire, understand, and apply basic math facts. Remedial and Special Education, 13(3), 19-35.

Miller, S.P. (2009). Validated Practices for Teaching Students with Diverse Needs and Abilities. 2nd edition. Upper Saddle River: New Jersey.

Morin, V. A., \& Miller, S. P. (1998). Teaching multiplication to middle school students with mental retardation. Education and Treatment of Children, 21(1), 22-36.

National Mathematics Advisory Panel. (2008). Foundations for success: The final report of the national mathematics advisory panel. Washington, DC: U.S. Department of Education.

Peterson, S. K., Mercer, C. D., \& O'Shea, L. (1988). Teaching learning disabled students place value using the concrete to abstract sequence. Learning Disabilities Research, 4, 52-56.

Rivera-Batiz, F. L. (1992). Quantitative literacy and the likelihood of employment among young adults in the United States. The Journal of Human Resources, 27, 313-328.

Strozier, S. D., Flores, M. M., Shippen, E. M., Hinton, V. (2012). The effects of concrete-representationalabstract sequence and a mnemonic strategy on algebra skills of students who struggle in math. Unpublished doctoral dissertation. Auburn, AL: Auburn University.

Witzel, B. S. (2005). Using cra to teach algebra to students with math difficulties in inclusive settings. Learning Disabilities: A Contemporary Journal 3, 49-60.

Witzel, B. S., Mercer, C. D., \& Miller, M. D. (2003). Teaching algebra to students with learning difficulties in inclusive setting. Learning Disabilities: A Contemporary Journal, 3(2), 49-60. 